\title{
Associations between Sarcopenia and Metabolic Risk Factors: A Systematic Review and Meta-Analysis
}

\author{
Yang Du, Chorong Oh, Jaekyung No* \\ Department of Food and Nutrition, Kyungsung University, Busan, Korea
}

Background: Metabolic risk factors can impact sarcopenia, but the direct relationship of metabolic risk factors with sarcopenia has not been examined. Our purpose was to investigate the effects of metabolic risk factors on sarcopenia in older adults.

Methods: Sixteen studies were found through a search of electronic databases and were subjected to a metaanalysis to investigate the differences in metabolic risk factors between patients with sarcopenia and controls. The random-effects standardized mean difference $\pm 95 \%$ confidence interval was calculated as the effect size. Results: The results showed that body mass index (BMI), fasting glucose, systolic blood pressure (SBP), diastolic blood pressure (DBP), triglycerides (TG), homeostasis model assessment of insulin resistance (HOMA-IR), highdensity lipoprotein cholesterol (HDL-C), and total cholesterol $(\mathrm{d}=3.252, \mathrm{~d}=2.039, \mathrm{~d}=2.956, \mathrm{~d}=2.579, \mathrm{~d}=2.123$, $d=1.195, d=-0.991$, and $d=1.007$, respectively) all had relationships with sarcopenia. In addition, the effect sizes of all male groups for all variables were higher than those of the female groups. However, only the betweensex effect size of HOMA-IR $(P<0.01)$ was significant, while those for BMl, fasting glucose, SBP, DBP, TG, HDL-C, low-density lipoprotein cholesterol, and total cholesterol were not. Finally, the metabolic risk factors appeared to be significantly related to loss of skeletal muscle.

Conclusion: Nutrition and appropriate exercise to enhance muscle strength and quality in the elderly reduce the occurrence of sarcopenia, thereby reducing the incidence of metabolic diseases.

Key words: Sarcopenia, Metabolic diseases, Risk factors, Meta-analysis, Aged

\author{
Received March 26, 2018 \\ Reviewed May 18, 2018 \\ Accepted July 30, 2018 \\ *Corresponding author \\ Jaekyung No \\ (iD) \\ https://orcid.org/0000-0003-2433-346X \\ Department of Food and Nutrition, \\ Kyungsung University, 309 Suyeong-ro, \\ Nam-gu, Busan 48434, Korea \\ Tel: +82-51-663-4651 \\ Fax: +82-51-663-4651 \\ E-mail:jkno3@ks.ac.kr
}

\section{INTRODUCTION}

The World Health Organization has reported that the global population of people older than the age of 65 years will reach at least 2 billion by $2050 .^{1}$ The aging process can lead to sarcopenia, metabolic diseases, and other chronic diseases. According to the latest annual report on causes of death in the elderly in Korea, the number of patients with sarcopenia is rapidly increasing among those older than 65 years. Sarcopenia is considered to be a common cause of mortality in this age group. ${ }^{2}$ In particular, sarcopenia has an increasing impact on the elderly, including incidence ${ }^{3}$, dis- ability ${ }^{4}$, health management costs ${ }^{5}$, and mortality. ${ }^{6}$ Therefore, sarcopenia is one of the major public health concerns among Korean older adults. It is suggested that the whole world should work together to cope with this health issue and prevent the occurrence of sarcopenia and sarcopenia-related complications (such as hypertension and diabetes) in old age.

Sarcopenia is a syndrome associated with impaired muscle and metabolic function characterized by an age-related decline in skeletal muscle mass and low levels of muscle function (muscle strength and physical activity)..$^{7}$ A number of studies have found ${ }^{8-10}$ that the diagnostic criteria for sarcopenia mainly consist of systolic blood 
pressure (SBP), diastolic blood pressure (DBP), body mass index (BMI), and homeostasis model assessment of insulin resistance (HOMA-IR). Although sarcopenia working groups all over the world have introduced sarcopenic diagnostic criteria, sarcopenia is a relatively new concept, and assessment or diagnosis of the indicators is still controversial.

With the increase of the global elderly population, sarcopenia will be increasingly common. ${ }^{11}$ Sarcopenia is prone to increase the mortality rate of the elderly since it can increase the risk of metabolic diseases. ${ }^{12}$ Metabolic diseases refer to clinical syndromes in which risk factors for multiple cardiovascular diseases such as obesity, hypertension, hyperglycemia, dyslipidemia, and the like coexist in an individual. Insulin resistance is the basis of this clinical syndrome. Recent studies reported that decreased skeletal muscle mass increases insulin resistance in vivo, which is closely related to the occurrence of metabolic diseases. ${ }^{13,14}$ For these reasons, increasing body mass can improve insulin sensitivity. ${ }^{15}$ In addition, sarcopenia results in atherosclerosis and triggers high blood pressure. ${ }^{16}$ With aging, body composition changes, loss of skeletal muscle, and/or increased fat mass may increase the risk of functional impairment and chronic metabolic disease.

Therefore, we performed a meta-analysis of the literature to determine the relationship between sarcopenia and possible metabolic risk factors. The objective of this study was to identify early-stage metabolic risk factors for sarcopenia. The results of this study should support instrumental suggestions for medical institutions and convalescent organizations to carry out corresponding preventive nutrition interventions to reduce the occurrence of metabolic diseases as early as possible.

\section{METHODS}

Although a meta-analysis is not a primary research method, it does include steps such as formulation of a problem, collection of data (studies), coding of data, and data analysis and interpretation. ${ }^{17}$

\section{Search strategy}

Two investigators (YD, JK No) independently conducted an electronic literature search of papers published from January 1, 1989 to
September 1, 2017. They conducted a thorough search of the four Korean electronic databases, KMbase, KISS, NDSL, and RISS; and of three overseas databases, PubMed, ScienceDirect, and Cochrane Library. For the PubMed search, controlled vocabulary terms and the following keywords were used: ("Sarcopenia" $[\mathrm{MeSH}]$ OR Sarcopenia [Title/Abstract]) AND ("Metabolic Diseases"[MeSH]) OR (Metabolic Diseases [Title/Abstract]) OR (Thesaurismosis [Title/Abstract]) OR (Thesaurismoses [Title/Abstract]) OR (Diseases, Metabolic [Title/Abstract]) OR (Disease, Metabolic [Title/ Abstract]) OR (Metabolic Disease [Title/Abstract]) and similar search strategy was run in other terms, which was restricted to studies published in English or Korean. In addition, the systematic identification, approval, synthesis, statistical merging, and reporting of the entire process of data extraction and selected studies were conducted based on a systematic review and meta-analysis of the $\mathrm{Na}$ tional Evidence-based Healthcare Collaborating Agency. ${ }^{18}$

\section{Study selection}

We included studies that (1) compared data on metabolic risk factors between participants with sarcopenia versus those without, (2) reported on metabolic risk factors such as BMI, fasting glucose, SBP, DBP, triglycerides (TG), HOMA-IR, high-density lipoprotein cholesterol (HDL-C), low-density lipoprotein cholesterol (LDLC), and total cholesterol, and (3) separated the data from men and women. Studies were excluded if they (1) did not measure or did not report metabolic risk factors in both sarcopenia and no sarcopenia subjects, (2) examined subjects younger than 65 years or used animal models, or (3) did not measure or report metabolic risk factors both in patients with and without sarcopenia.

\section{Data extraction}

Two authors (YD, JK No) independently extracted data from the selected studies into a standardized Microsoft Excel spreadsheet. Any disagreement was resolved by consensus. The following information was extracted: (1) study population characteristics (e.g., sample size, demographic), (2) survey site at which the study was performed, (3) parameters related to metabolic risk factors in individuals with sarcopenia versus no sarcopenia, and (4) compared data from men and women separately from the overall population. 


\section{Quality assessment}

In meta-analyses, the "file drawer problem" refers to unknown, unpublished research whose results fail to confirm the pattern revealed by the published findings. ${ }^{19}$ If no unpublished research is retrieved, a publication bias can exist in favor of significant findings, which could distort the results of the meta-analysis. Cooper $^{20} \mathrm{de}-$ veloped a method for determining the magnitude of the file drawer problem: calculating the minimum number of unpublished studies reporting not significant findings that would be necessary to overturn the conclusion reached in a particular meta-analysis. This number has been defined as the fail-safe number $(\mathrm{Nfs}) .^{20}$ Rosenthal and Hall ${ }^{21}$ have proposed that a reasonable tolerance level of the file drawer problem has been achieved if the Nfs exceeds $5 n+10$ (n, number of studies included in the meta-analysis).

\section{Statistical analysis}

The meta-analysis was performed using comprehensive metaanalysis V2.0 for Windows (https://www.meta-analysis.com/). Only outcomes from at least two studies can be subjected to metaanalysis, while outcomes from only one study were reported in the descriptive analyses. When combining studies, the random effects model was used to account for study heterogeneity ${ }^{22}$ by utilizing the standardized mean difference with its $95 \%$ confidence interval (CI). Study heterogeneity was measured using the chi-square and I-square statistics, with chi-square $P \leq 0.05$ and I-square $\geq 50 \%$ indicating the presence of crucial heterogeneity. Publication bias was assessed with a visual inspection of funnel plots and the Egger bias test ${ }^{23}$ for outcomes within these metabolic risk factors. Furthermore, this study also utilized Nfs to verify the reliability of the researched nine metabolic risk factors. These factors were used for subgroup analysis based on the analysis of included studies, and the subgroup analysis compared men and women.

\section{RESULTS}

The search identified 991 potentially eligible studies, of which 384 duplicates were excluded. After excluding 547 papers through title and abstract review, 60 full-text articles were examined. After further examination, 16 studies were included in the meta-analysis (Fig. 1).2439

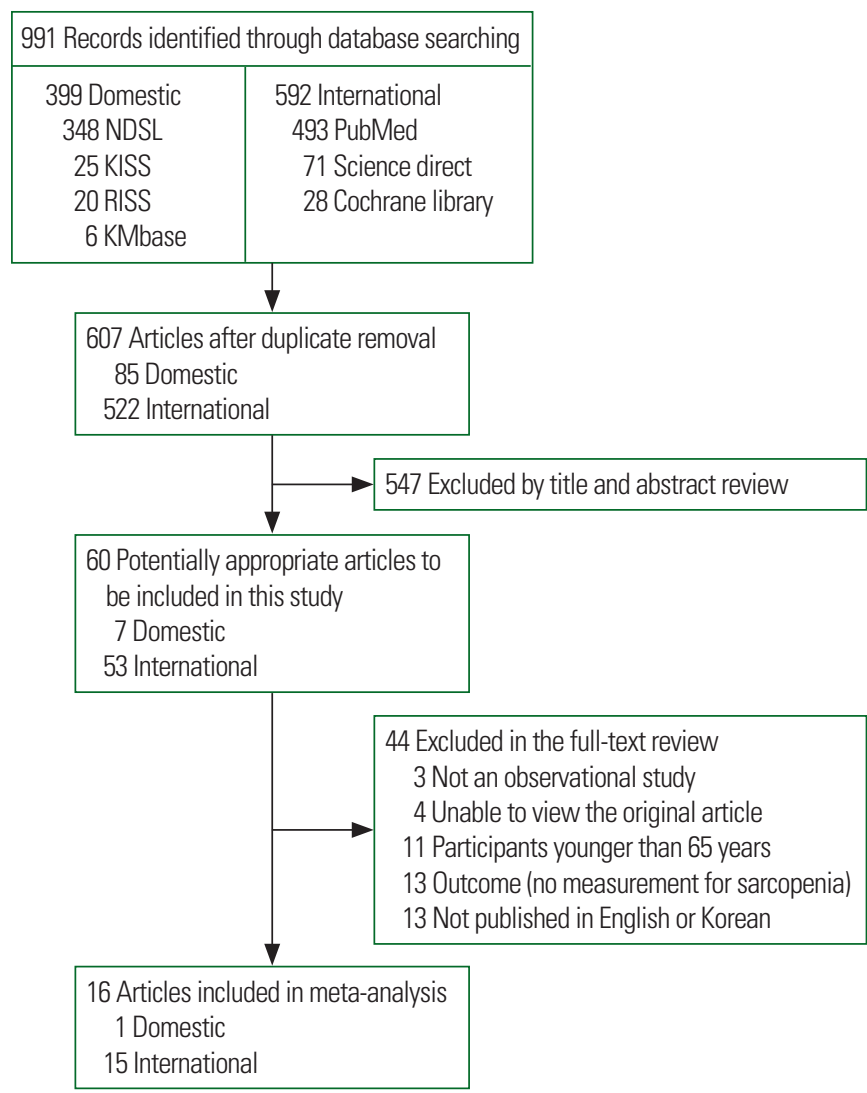

Figure 1. Flow of study analysis through different phases of the meta-analysis (from January 1, 1989 to September 1, 2017).

\section{Study and patient characteristics}

Study and patient characteristics are summarized in Table 1 . The 16 meta-analyzed studies included a total of 803,022 participants (62,273 with sarcopenia and 740,749 without). The majority of the studies were conducted in Asia and by social survey. All of the studies were published after the year 2000 . The ages of the subjects ranged from 65 to 70 years in 11 papers $(68.75 \%)$ and from 70 to 80 years in 10 papers $(62.25 \%)$, and there were five papers in which the ages ranged from 65 to 80 years. There were two papers (12.5\%) on studies of elderly women, four papers $(25 \%)$ on elderly men, and 10 papers $(62.5 \%)$ on both men and women. Among the 16 studies for the meta-analysis, the numbers of instances of the use of BMI, fasting glucose, SBP, DBP, TG, HOMA-IR, HDL-C, LDL-C, and total cholesterol as adjusted variables were 27, 20, 9, 9, $14,12,12,7$, and 14 , respectively, since there were differences between the male and female groups. 
Table 1. Characteristics of the included studies

\begin{tabular}{|c|c|c|c|c|}
\hline Author (year), country & $\begin{array}{c}\text { Sample size } \\
\text { (with/without sarcopenia) }\end{array}$ & $\operatorname{Sex}(\%)$ & Setting & Adjusted variable \\
\hline \multirow[t]{2}{*}{ Lee et al. $(2013)^{24}$, South Korea } & $1,535(510 / 1,025)$ & M: 54.30 & Social & BMI, fasting glucose, HOMA-IR \\
\hline & & $F: 45.70$ & & \\
\hline \multirow[t]{2}{*}{ Choi and Park $(2016)^{25}$, South Korea } & $780,994(57,246 / 723,748)$ & M: 52.85 & Social & BMI, fasting glucose, SBP, DBP, TG, HDL-C, total cholesterol \\
\hline & & F: 47.15 & & \\
\hline Kang et al. $(2017)^{27}$, South Korea & $2,628(557 / 2,071)$ & F: 100 & Social & BMI, fasting glucose, SBP, DBP, TG, HDL-C, total cholesterol \\
\hline \multirow[t]{2}{*}{ Chung et al. $(2013)^{28}$, South Korea } & $2,943(1,248 / 1,695)$ & M: 42.47 & Social & BMI, fasting glucose, SBP, DBP, TG, HOMA-IR, HDL-C, LDL-C, total cholesterol \\
\hline & & F: 57.53 & & \\
\hline Isanejad et al. (2016)29, Finland & $496(127 / 369)$ & F: 100 & Community & $\mathrm{BMl}$ \\
\hline \multirow[t]{2}{*}{ Kim et al. $(2014)^{30}$, South Korea } & $2,264(540 / 1,724)$ & M: 41.52 & Social & BMI, TG, HOMA-IR, HDL-C, LDL-C, total cholesterol \\
\hline & & F: 58.48 & & \\
\hline \multirow[t]{2}{*}{ Buchmann et al. (2016) $)^{31}$, Germany } & $1,402(280 / 1,122)$ & M: 51.07 & Community & BMI, fasting glucose, TG, HOMA-IR, HDL-C \\
\hline & & F: 48.93 & & \\
\hline \multirow[t]{2}{*}{ Lim et al. $(2010)^{35}$, South Korea } & $565(235 / 330)$ & M: 50.80 & Social & BMI, fasting glucose, TG, HOMA-IR, HDL-C, LDL-C, total cholesterol \\
\hline & & $F: 49.20$ & & \\
\hline \multirow[t]{2}{*}{ Chalhoub et al. $(2015)^{34}$, United States } & $3,802(127 / 3,675)$ & M: 90.64 & Community & $\mathrm{BMl}$ \\
\hline & & F: 9.36 & & \\
\hline Pereira et al. $(2015)^{33}$, Brazil & $173(20 / 153)$ & M: 100 & Social & $\mathrm{BMl}$ \\
\hline \multirow[t]{2}{*}{ Ishii et al. (2014)³2, Japan } & $1,971(359 / 1,612)$ & M: 49.57 & Social & $\mathrm{BMl}$ \\
\hline & & F: 50.43 & & \\
\hline Kim et al. $(2017)^{26}$, South Korea & $435(138 / 297)$ & M: 100 & Social & BMI, fasting glucose, SBP, DBP, TG, HDL-C,LDL-C, total cholesterol \\
\hline \multirow[t]{2}{*}{ Baumgartner $(2000)^{36}$, United States } & $562(216 / 346)$ & M: 51.33 & Community & BMI, fasting glucose, total cholesterol \\
\hline & & F: 48.67 & & \\
\hline Chin et al. $(2013)^{37}$, South Korea & $1,076(176 / 900)$ & M: 100 & Social & BMI, fasting glucose, TG, HOMA-IR, HDL-C, LDL-C, total cholesterol \\
\hline \multirow[t]{2}{*}{ Moon et al. $(2015)^{38}$, South Korea } & $674(35 / 639)$ & M: 47.16 & Social & BMI, fasting glucose, SBP, DBP, TG, HDL-C \\
\hline & & F: 52.84 & & \\
\hline Han et al. (2014) $)^{39}$, South Korea & $1,502(459 / 1,043)$ & M: 100 & Social & BMI, fasting glucose, SBP, DBP, TG, HOMA-IR, HDL-C, total cholesterol \\
\hline
\end{tabular}

M, male; F, female; BMI, body mass index; HOMA-IR, homeostasis model assessment of insulin resistance; SBP, systolic blood pressure; DBP, diastolic blood pressure; TG, triglycerides; HDL-C, high-density lipoprotein cholesterol; LDL-C, low-density lipoprotein cholesterol.

Table 2. Summary of results, overall effect sizes, and homogeneity of d-value

\begin{tabular}{|c|c|c|c|c|c|c|}
\hline \multirow{2}{*}{ Outcome } & \multirow{2}{*}{ Number* } & \multirow{2}{*}{$\frac{\mathrm{d}^{\dagger}(95 \% \mathrm{Cl})}{\text { Random-effect }}$} & \multicolumn{3}{|c|}{ Homogeneity of d-value } & \multirow{2}{*}{ Nfs } \\
\hline & & & $0^{\S}$ & $P^{2}(\%) \|$ & $P \rrbracket$ & \\
\hline $\mathrm{BMl}$ & 27 & 3.252 (2.657 to 3.847 ) & $43,587.486$ & 99.940 & 0.0000 & 5,668 \\
\hline DBP & 9 & 2.579 (1.066 to 4.091$)$ & $74,498.836$ & 99.989 & 0.0000 & 14,903 \\
\hline Fasting glucose & 20 & 2.039 (1.078 to 3.000$)$ & $81,578.937$ & 99.977 & 0.0000 & 740 \\
\hline HDL-C & 16 & -0.991 (-2.081 to 0.099) & 93,333.099 & 99.984 & 0.0000 & 5,915 \\
\hline HOMA-IR & 12 & 1.195 (0.481 to 1.910$)$ & $2,427.413$ & 99.547 & 0.0000 & 2,628 \\
\hline LDL-C & 7 & $0.144(-0.131$ to 0.419$)$ & 114.811 & 94.774 & 0.0000 & 21 \\
\hline SBP & 9 & 2.956 (2.316 to 3.597 ) & $13,336.577$ & 99.940 & 0.0000 & 608 \\
\hline TG & 14 & 2.123 (0.542 to 3.704 ) & $155,743.065$ & 99.992 & 0.0000 & 9,786 \\
\hline Total cholesterol & 14 & 1.007 (-0.914 to 2.928$)$ & $226,491.621$ & 99.994 & 0.0000 & 642 \\
\hline
\end{tabular}

${ }^{*}$ The number of adjusted variables; ${ }^{\dagger}$ Overall effect size; ${ }^{\ddagger}$ Indicates a significant effect $(P<0.001)$; ${ }^{\S}$ Cochran's 0 indicating significance of heterogeneity; "The magnitude of heterogeneity; " $P$-value represents the significance of heterogeneity.

$\mathrm{Cl}$, confidence interval; Nfs, fail-safe number; BMI, body mass index; DBP, diastolic blood pressure; HDL-C, high-density lipoprotein cholesterol; HOMA-IR, homeostasis model assessment of insulin resistance; LDL-C, low-density lipoprotein cholesterol; SBP, systolic blood pressure; TG, triglycerides. 


\section{Effect sizes}

After data from the accepted 16 studies were pooled, all nine metabolic risk factors of interest were found to have a significant relationship with sarcopenia (Table 2), and the corresponding forest plots of these nine effect sizes are demonstrated in Fig. 2. Overall effect sizes under random-effects assumptions indicate that BMI $(\mathrm{d}=3.252$; 95\% CI, 2.657-3.845; $P<0.001)$, fasting glucose $(\mathrm{d}=$ 2.039; 95\% CI, 1.078-3.000; $P<0.001)$, SBP ( $d=2.956$; 95\% CI, 2.316-3.579; $P<0.001)$, DBP $(\mathrm{d}=2.579 ; 95 \% \mathrm{CI}, 1.066-4.091$; $P<0.001)$, TG $(\mathrm{d}=2.123$; 95\% CI, 0.542-3.704; $P<0.001)$,

\section{A}

Study name Std diff in means and $95 \% \mathrm{Cl}$

Lee et al. $M$
Choi et al. M

Kang et al. M

Kim et al. $M$

Buchmann et al. $M$
Lim et al $M$

Lim et al. $M$

Pereira et al. $M$

shii et al. M

Yun $M$

Chin et al. $M$

Han et al. $M$

Lee et al. $F$

Choi et al. $F$

Kang et al. $F$

Chung et al. $F$

Isanejad et al.
Kim et al. F

Buchmann et al. $F$

Lim et al. F

shii et al. $F$

Baumgartner $F$
Moon et al. $F$
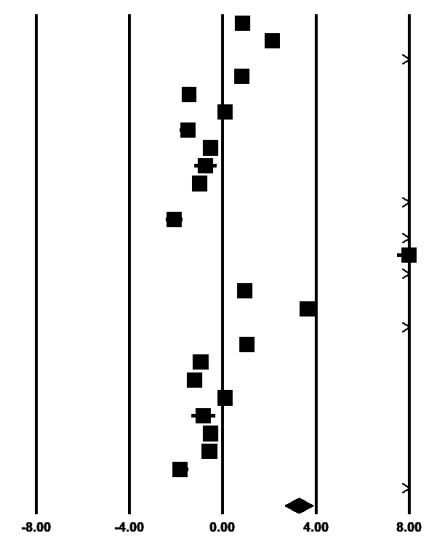

\section{B}

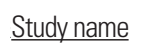

Std diff in means and 95\% $\mathrm{Cl}$

Lee et al. $M$ Choi et al. $M$ Kim et al. M Kim et al. M Buchmann et al. $M$ Yun $M$ Yun $\mathrm{M}$
Baumgartner $\mathrm{M}$ Chin et al. M Moon et al. M Han et al. M Lee et al. $F$ Choi et al. $F$ Kang et al. $F$ Kimg a. Kim etal. $F$ Buchmann et Baumgartner $F$ Moon et al. $F$
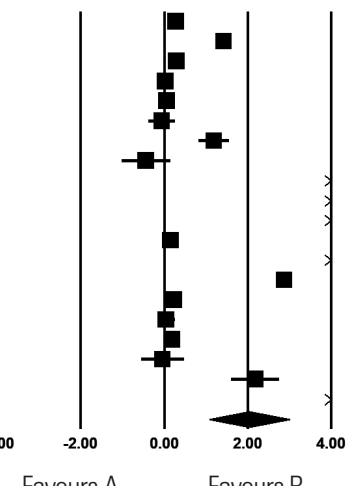

Favours A

Favours B

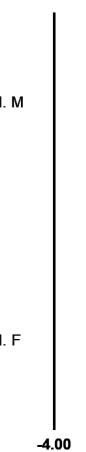

C

Study name $\underline{\text { Std diff in means and } 95 \% \mathrm{Cl}}$

Favours A

Favours B

(D)

Study name

Choi et al. M

Chung et al. M

Yun $M$

Moon et al. M

Han et al. M

Choi et al. F

Kang et al. $F$

Chung et al. F

Moon et al. F

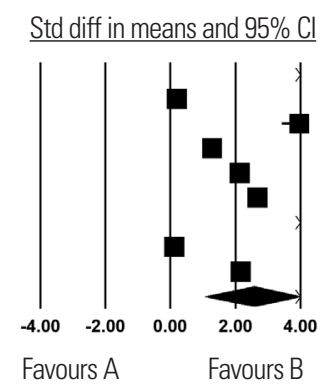

E

Study name

\section{Choi et al. M} Kim et al. M Buchmann et al. M Lim et al. M

Yun $\mathrm{M}$

Chin et al. M

Moon et al. M

Han et al. M

Choi et al. F

Kang et al. $F$

Kim et al. F

Buchmann et al. $F$

Lim et al. F

Moon et al. F
Choi et al. M Chung et al. $\mathbf{M}$ Yun M Moon et al. M Han et al. M Choi et al. F Kang et al. F Chung et al. F Moon et al. F

F

Study name

Lee et al. M Chung et al. M Kim et al. M Buchmann et al. M Lim et al. M Chin et al. M

Han et al. M

Lee al. $F$

Chung et al. F

Kim et al. F

Buchmann et al. $F$

Lim et al. F

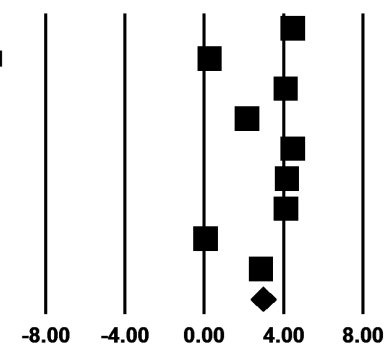

Favours A

Favours B

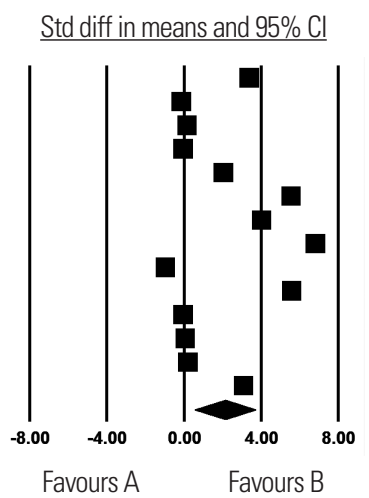

Favours A

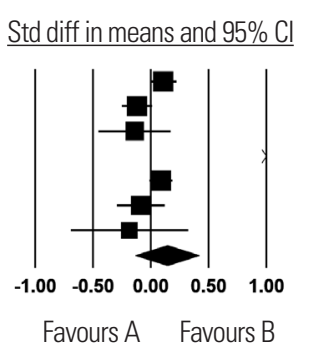

G

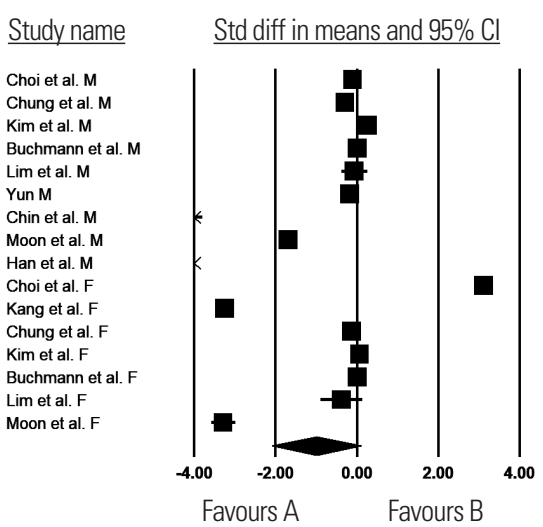

(H)

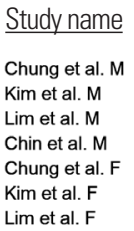

Study name

ung et al. Lim et al. $M$ Chung et al. im et al. F
(1)

Study name

Choi et al. M Chung et al. M Kim et al. M Lim et al. M Yun $M$

Baumgartner $\mathrm{M}$ Chin et al. M

Han et al. M

Choi et al. F Kang et al. $\mathrm{F}$ Chung et al. F Kim et al. $F$ Lim et al. $F$ Baumgartner $F$ $\underline{\text { Std diff in means and } 95 \% \mathrm{Cl}}$

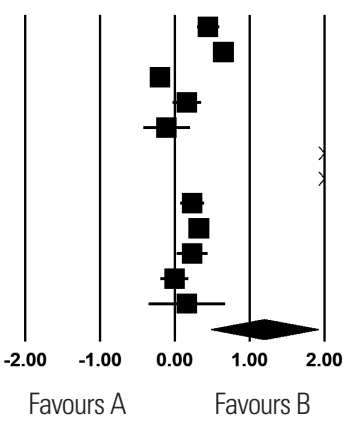

Std diff in means and $95 \% \mathrm{Cl}$

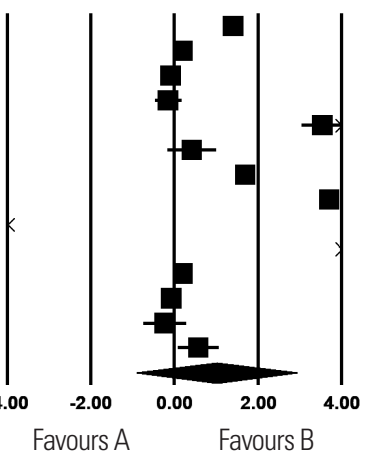

Figure 2. Forest plots of (A) body mass index, (B) fasting glucose, (C) systolic blood pressure, (D) diastolic blood pressure, (E) triglycerides, (F) homeostasis model assessment of insulin resistance, $(G)$ high-density lipoprotein cholesterol, $(H)$ low-density lipoprotein cholesterol, and (I) total cholesterol in subjects with sarcopenia vs. without sarcopenia. Std diff, standard difference; Cl, confidence interval; $\mathrm{M}$, male; F, female. 
Table 3. Effect sizes by sex

\begin{tabular}{|c|c|c|c|c|c|c|}
\hline Outcome & Subgroup & Number* & $\mathrm{d}^{\dagger}(95 \% \mathrm{Cl})$ & $Q^{\ddagger}$ & $1^{2}(\%)^{\S}$ & $P \|$ \\
\hline \multirow[t]{2}{*}{$\mathrm{BMl}$} & Male & 15 & 4.317 (3.027 to 5.608) & $13,489.344$ & 99.896 & 0.071 \\
\hline & Female & 12 & 2.409 (0.792 to 4.025) & $16,651.096$ & 99.934 & \\
\hline \multirow[t]{2}{*}{ DBP } & Male & 5 & $2.693(-0.289$ to 5.675$)$ & $13,871.865$ & 99.971 & 0.883 \\
\hline & Female & 4 & 2.437 (0.806 to 4.068) & 3,320.932 & 99.910 & \\
\hline \multirow[t]{2}{*}{ Fasting glucose } & Male & 11 & 2.268 (1.379 to 3.157$)$ & 4,323.045 & 99.769 & 0.610 \\
\hline & Female & 9 & $1.75(-0.031$ to 3.531$)$ & $15,143.460$ & 99.947 & \\
\hline \multirow[t]{2}{*}{ HDL-C } & Male & 9 & $-1.334(-2.172$ to -0.496$)$ & $3,087.016$ & 99.741 & 0.527 \\
\hline & Female & 7 & $-0.546(-2.841$ to 1.749$)$ & $16,712.701$ & 99.964 & \\
\hline \multirow[t]{2}{*}{ HOMA-IR } & Male & 7 & 1.933 (0.665 to 3.201) & $2,270.527$ & 99.736 & $0.008 \pi$ \\
\hline & Female & 5 & 0.209 (0.083 to 0.335) & $9.694^{* *}$ & 58.739 & \\
\hline \multirow[t]{2}{*}{ LDL-C } & Male & 4 & $0.274(-0.232$ to 0.780$)$ & 109.277 & 97.255 & 0.336 \\
\hline & Female & 3 & $0.051(-0.035$ to 0.137$)$ & $3.096^{* *}$ & 35.396 & \\
\hline \multirow[t]{2}{*}{ SBP } & Male & 5 & 3.093 (0.973 to 5.213) & $5,610.668$ & 99.929 & 0.855 \\
\hline & Female & 4 & 2.801 (0.504 to 5.099) & 6,791.894 & 99.956 & \\
\hline \multirow[t]{2}{*}{ TG } & Male & 8 & 2.723 (1.159 to 4.286) & $5,287.473$ & 99.868 & 0.319 \\
\hline & Female & 6 & 1.322 (0.940 to 3.585$)$ & $6,156.489$ & 99.919 & \\
\hline \multirow[t]{2}{*}{ Total cholesterol } & Male & 8 & 1.337 (0.583 to 2.091) & $1,830.212$ & 99.618 & 0.645 \\
\hline & Female & 6 & 0.562 (-2.648 to 3.773$)$ & $19,166.840$ & 99.974 & \\
\hline
\end{tabular}

${ }^{*}$ The number of adjusted variables; ' ${ }^{E}$ ffect size; ${ }^{\ddagger}$ Cochran's $Q$ indicating significance of heterogeneity; ${ }^{\text {TT}}$ The magnitude of heterogeneity; $" P$-value represents the significance of heterogeneity; "Indicates a significant effect $(P<0.01) ;{ }^{* *}$ Indicates fixed-effects.

$\mathrm{Cl}$, confidence interval; BMI, body mass index; DBP, diastolic blood pressure; HDL-C, high-density lipoprotein cholesterol; HOMA-IR, homeostasis model assessment of insulin resistance; LDL-C, low-density lipoprotein cholesterol; SBP, systolic blood pressure; TG, triglycerides.

HOMA-IR ( $\mathrm{d}=1.195$; 95\% CI, 0.481-1.910; $P<0.001$ ), HDL-C $(\mathrm{d}=-0.991 ; 95 \% \mathrm{CI},-2.08$ to $0.099 ; P<0.0001)$, LDL-C ( $=$ $0.144 ; 95 \% \mathrm{CI},-0.131$ to $0.419 ; P<0.001)$, and total cholesterol ( $\mathrm{d}=1.007 ; 95 \% \mathrm{CI},-0.914$ to $2.928 ; P<0.001$ ) had a significant overall effect on sarcopenia. There was a large degree of heterogeneity among studies, with $\mathrm{I}^{2}$ ranging from $99.547 \%$ to $99.994 \%$.

\section{Subgroup analysis}

The results of the random-effects categorical analysis by male and female subgroups are illustrated in Table 3 . The results for the relationship of sarcopenia with metabolic risk factors in men and women were as follows: (1) the effect sizes of all of the male groups were higher than those of the female groups; (2) however, only the effect size of HOMA-IR $(P<0.01)$ was significant, while the differences of the effect sizes between men and women within each of the other eight risk factor subgroups, namely BMI, fasting glucose, SBP, DBP, TG, HDL-C, LDL-C, and total cholesterol, were not.

\section{Reliability test}

The Nfs computed for this meta-analysis regarding the effects of
BMI, fasting glucose, SBP, DBP, TG, HOMA-IR, HDL-C, LDL-C, and total cholesterol on sarcopenia were 5,668, 740, 608, 14,903, $9,786,2,628,5,915,21$, and 642 unpublished studies, respectively (Table 2). Only in the case of LDL-C were the Nfs not exceeded, with 45 unpublished studies; therefore, it is difficult to support the effect size.

\section{Publication bias}

Publication bias was evaluated to examine the validity of the results of this study. The effect size of the included studies was not visually symmetrical in the funnel plot, which is illustrated in Fig. 3. An Egger linear regression test inferred the severity of the publication bias. ${ }^{40}$ As a result, we added no new studies to convert the effect size of the included studies from asymmetry to symmetry. Therefore, the pooled effect size did not convert. To sum up, we could not ensure that the included studies had no publication bias; however, there was also no evidence to call the validity of the results into question. 
D

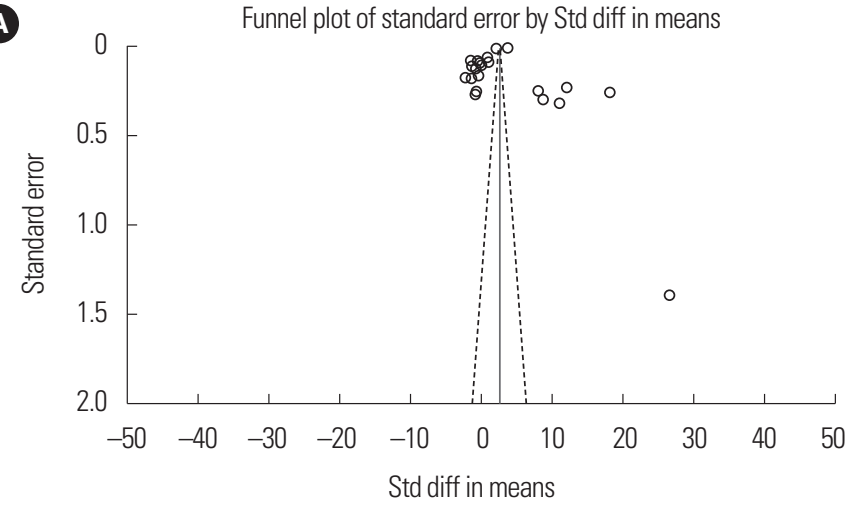

○

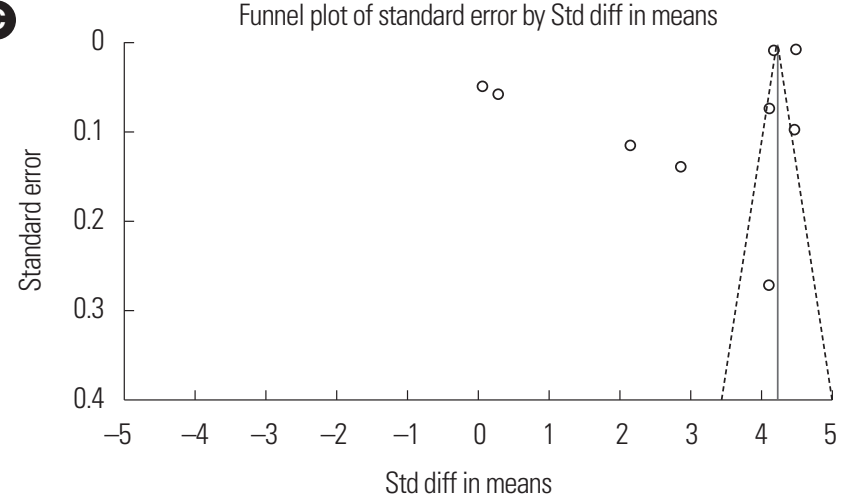

E

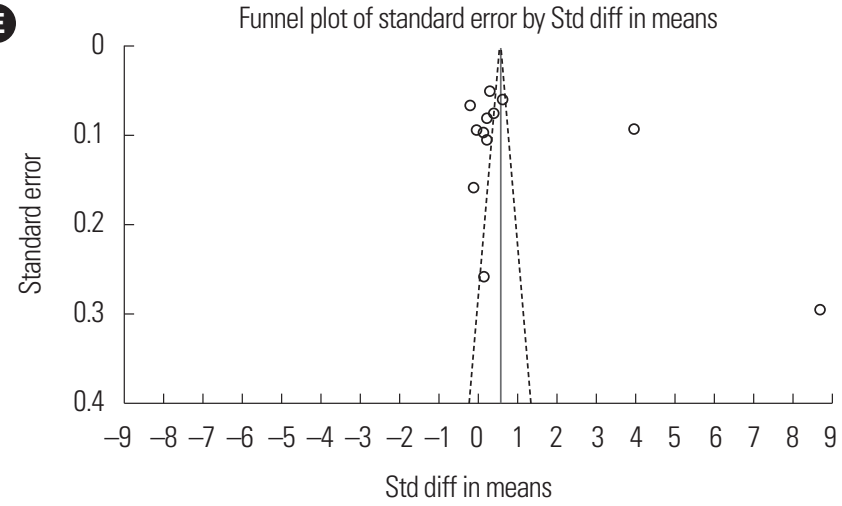

G

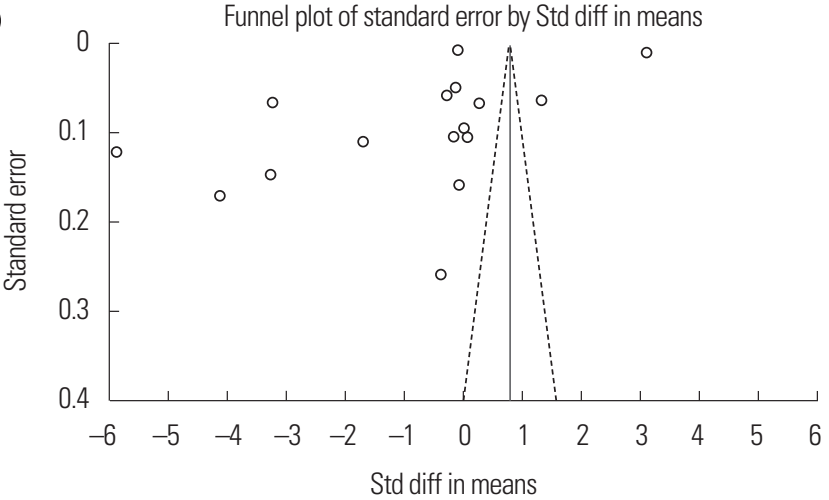

B

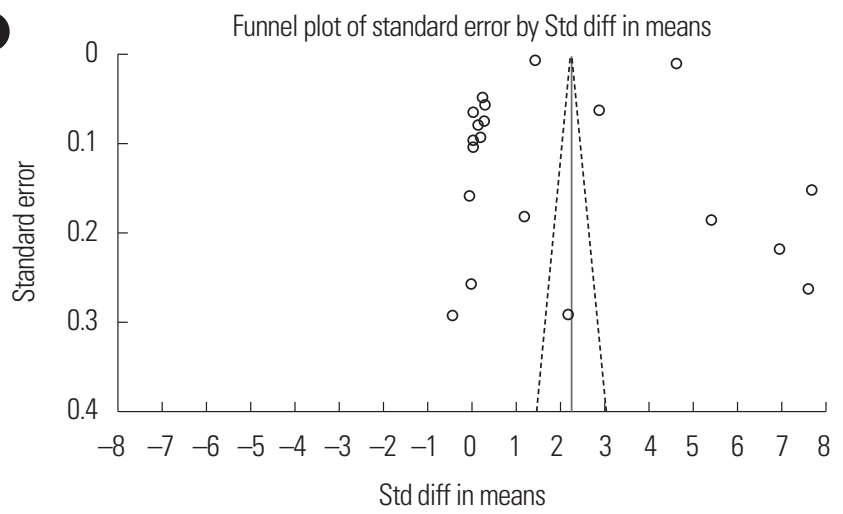

(1)

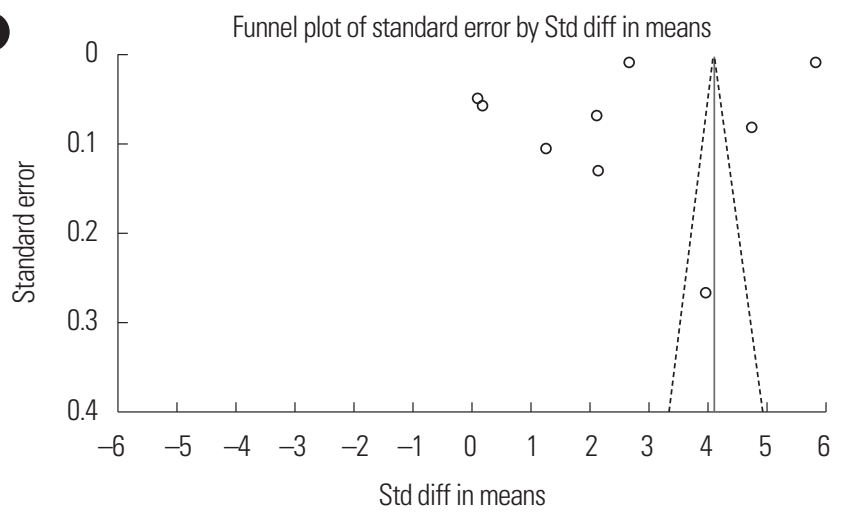

•

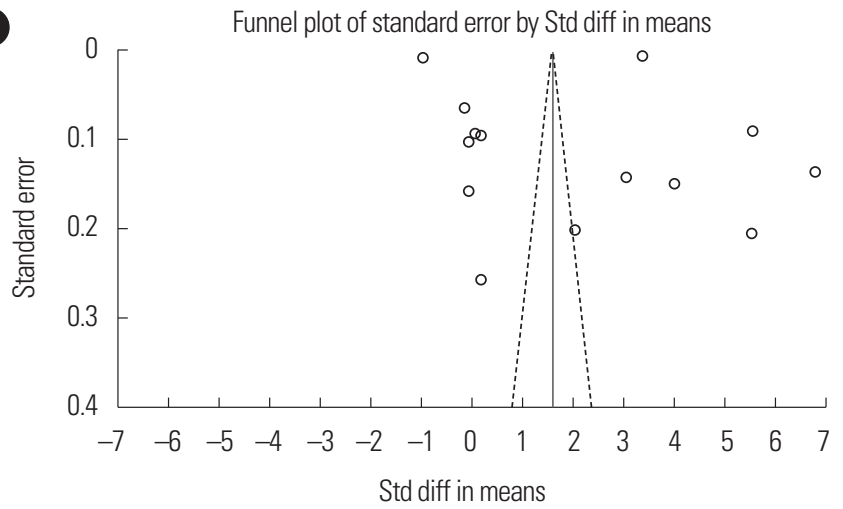

(十)

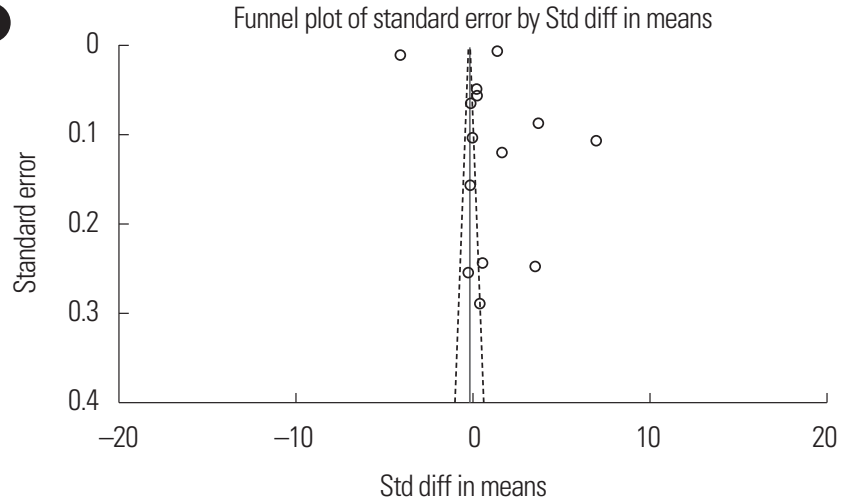

Figure 3. Funnel plots of (A) body mass index, (B) fasting glucose, (C) systolic blood pressure, (D) diastolic blood pressure, (E) triglycerides, (F) homeostasis model assessment of insulin resistance, (G) high-density lipoprotein cholesterol, and (H) total cholesterol. Std diff, standard difference. 


\section{DISCUSSION}

In this meta-analysis involving 62,273 people with sarcopenia and 740,749 without, we found that the nine metabolic risk factors (BMI, fasting glucose, SBP, DBP, TG, HOMA-IR, HDL-C, LDL$\mathrm{C}$, and total cholesterol) investigated are related to sarcopenia. Although LDL-C had a significant effect size, the reliability test of LDL-C showed that the results of the research did not support its effect size. Therefore, we will conduct more detailed and in-depth studies on the effects of LDL-C on sarcopenia. To some extent, other factors may be associated with sarcopenia, which we will examine in future research; for example, body fat percentage, waist circumference, and visceral fat area. To the best of our knowledge, this is the first meta-analysis to investigate the possible relationship between sarcopenia and metabolic risk factors. The findings of this study on the relationship of metabolic risk factors with sarcopenia parameters, as reported in previous papers, complement the development of this research focus and provide instrumental details and statistics for a future study.

Several recently published systematic reviews on the relationship of metabolic risk factors with sarcopenia reported similar effects with $\mathrm{BMI}^{41}$, fasting glucose ${ }^{42,43}, \mathrm{SBP}$ and $\mathrm{DBP}^{44}, \mathrm{TG}^{45}, \mathrm{HOMA}$ $\mathrm{IR}^{41,46}, \mathrm{HDL}-\mathrm{C}$, and total cholesterol. ${ }^{45}$ Although the analysis investigating BMI, fasting glucose, SBP, DBP, TG, HOMA-IR, HDL-C, and total cholesterol as outcomes was characterized by high heterogeneity, we explained the majority of this with our meta regression analyses. According to Lu et al.s research ${ }^{14}$, low muscle mass and a form of obesity called sarcopenia are associated with metabolic syndrome in the American elderly. These findings are in agreement with our previous work ${ }^{41,46}$, in which BMI, fasting glucose, SBP, DBP, TG, HOMA-IR, HDL-C, and total cholesterol were more strictly related to sarcopenia compared with LDL-C. While the exact reason for this result is not clear, systematic reviews could provide an answer. Although our findings should be clarified and further explored with future longitudinal studies, our results support the notion that BMI, fasting glucose, SBP, DBP, TG, HOMA-IR, HDL-C, and total cholesterol could be used as parameters for detecting sarcopenia.

Finally, the studies did not adjust for any confounding variables, which may have affected both the exposure and outcome. ${ }^{47}$ Thus, adjusting for confounders is a good way to reduce potential bias. According to previous studies, sex can affect the correlation between metabolic risk factors and elder sarcopenia. ${ }^{41,46}$ Therefore, when we accumulate more results, we will perform a subgroup analysis. Moreover, our present subgroup analysis suggests that male sex plays an important role in explaining the association between metabolic risk factors and sarcopenia. This finding seems to be consistent with the current literature suggesting that men have higher metabolic risk factor levels compared with women. These findings suggest that, in the future, sarcopenia-preventive treatments should be sex specific. There are actually relatively few data directly addressing many of these points, all of which are important areas for future research.

This meta-analysis has several limitations. First, the number of included studies was insufficient. Second, while weight loss was not the objective in any of the included studies, we did not control for weight change among participants. Third, according to the criteria of the subgroup analysis, the study can be further refined if there are more heterogeneous samples. Last, ecological fallacy is a possibility as we did not have access to the raw data from the included studies, and we should therefore be cautious interpreting the group results as individual effects. Despite these limitations, to our knowledge, this is the first study to confirm the relationship between metabolic risk factors in sarcopenia in the elderly. We performed a comprehensive literature search using seven electronic databases. We performed moderation analysis on all variables, with sufficient data provided in the published material. Our research provides evidence for more effective and appropriate early preventive interventions and strategies to reduce the risk of metabolic diseases in the elderly. In the future, we will use a predictive model to calculate effect sizes for each significant moderator and transform that effect size into clinical units of measure for sarcopenia.

\section{CONFLICTS OF INTEREST}

The authors declare no conflict of interest.

\section{REFERENCES}

1. Beaudart C, Rizzoli R, Bruyère O, Reginster JY, Biver E. Sar- 
copenia: burden and challenges for public health. Arch Public Health 2014;72:45.

2. Hong S, Choi WH. Clinical and physiopathological mechanism of sarcopenia. Korean J Med 2012;83:444-54.

3. Sayer AA, Dennison EM, Syddall HE, Gilbody HJ, Phillips DI, Cooper C. Type 2 diabetes, muscle strength, and impaired physical function: the tip of the iceberg? Diabetes Care 2005; 28:2541-2.

4. Janssen I, Heymsfield SB, Ross R. Low relative skeletal muscle mass (sarcopenia) in older persons is associated with functional impairment and physical disability. J Am Geriatr Soc 2002; 50:889-96.

5. Janssen I, Shepard DS, Katzmarzyk PT, Roubenoff R. The healthcare costs of sarcopenia in the United States. J Am Geriatr Soc 2004;52:80-5.

6. Gale CR, Martyn CN, Cooper C, Sayer AA. Grip strength, body composition, and mortality. Int J Epidemiol 2007;36: 228-35.

7. Chen LK, Liu LK, Woo J, Assantachai P, Auyeung TW, Bahyah KS, et al. Sarcopenia in Asia: consensus report of the Asian Working Group for Sarcopenia. J Am Med Dir Assoc 2014; 15:95-101.

8. Trussell J, Henry N, Hassan F, Prezioso A, Law A, Filonenko A. Burden of unintended pregnancy in the United States: potential savings with increased use of long-acting reversible contraception. Contraception 2013;87:154-61.

9. Cruz-Jentoft AJ, Baeyens JP, Bauer JM, Boirie Y, Cederholm T, Landi F, et al. Sarcopenia: European consensus on definition and diagnosis: report of the European Working Group on Sarcopenia in Older People. Age Ageing 2010;39:412-23.

10. Chien MY, Huang TY, Wu YT. Prevalence of sarcopenia estimated using a bioelectrical impedance analysis prediction equation in community-dwelling elderly people in Taiwan. J Am Geriatr Soc 2008;56:1710-5.

11. World Health Organization. Ageing and life course [Internet]. Geneva: World Health Organization [cited 2009 Apr 30]. Available from: http://www.who.int/ageing/en/

12. Stenholm S, Harris TB, Rantanen T, Visser M, Kritchevsky SB, Ferrucci L. Sarcopenic obesity: definition, cause and consequences. Curr Opin Clin Nutr Metab Care 2008;11:693-700.
13. Lee J, Hong YP, Shin HJ, Lee W. Associations of sarcopenia and sarcopenic obesity with metabolic syndrome considering both muscle mass and muscle strength. J Prev Med Public Health 2016;49:35-44.

14. Lu CW, Yang KC, Chang HH, Lee LT, Chen CY, Huang KC. Sarcopenic obesity is closely associated with metabolic syndrome. Obes Res Clin Pract 2013;7:e301-7.

15. Solerte SB, Gazzaruso C, Bonacasa R, Rondanelli M, Zamboni M, Basso C, et al. Nutritional supplements with oral amino acid mixtures increases whole-body lean mass and insulin sensitivity in elderly subjects with sarcopenia. Am J Cardiol 2008; 101:69E-77E.

16. Sanada K, Miyachi M, Tanimoto M, Yamamoto K, Murakami $\mathrm{H}$, Okumura $\mathrm{S}$, et al. A cross-sectional study of sarcopenia in Japanese men and women: reference values and association with cardiovascular risk factors. Eur J Appl Physiol 2010;110: 57-65.

17. Rosenthal R. Parametric measures of effect size. In: Cooper H, Hedges LV, editors. The handbook of research synthesis. New York: Russell Sage Foundation; 1994. p. 231-44.

18. Kim G, Ahn J, Ha K, Lee CH, Woo JM, Lee JK, et al. National evidence-based collaborating agency (NECA) round-table conference consensus statement: multidisciplinary responses to suicide, the first ranked cause of death in adolescents. J Korean Med Assoc 2013;56:111-9.

19. Rosenthal R. The file drawer problem and tolerance for null results. Psychol Bull 1979;86:638-41.

20. Cooper HM. Statistically combining independent studies: a meta-analysis of sex differences in conformity research. J Pers Soc Psychol 1979;37:131-46.

21. Rosenthal R, Hall J. Critical values of $Z$ for combining independent probabilities. Replications Soc Psychol 1981;1:1-6.

22. Higgins JP, Thompson SG. Quantifying heterogeneity in a meta-analysis. Stat Med 2002;21:1539-58.

23. Duval S, Tweedie R. Trim and fill: a simple funnel-plot-based method of testing and adjusting for publication bias in metaanalysis. Biometrics 2000;56:455-63.

24. Lee SG, Lee YH, Kim KJ, Lee W, Kwon OH, Kim JH. Additive association of vitamin $\mathrm{D}$ insufficiency and sarcopenia with low femoral bone mineral density in noninstitutionalized el- 
derly population: the Korea National Health and Nutrition Examination Surveys 2009-2010. Osteoporos Int 2013;24: 2789-99.

25. Choi HA, Park KM. Effects of sarcopenic obesity on metabolic syndrome in Korean elders: using data from the Korea National Health and Nutrition Examination Survey (2008-2011). J Korean Acad Community Health Nurs 2016;27:231-41.

26. Kim JH, Ahn HJ, Yang TY, Kim SY. Sarcopenic obesity and associated factors in older adults with diabetes: the 2009-2010 Korean National Health and Nutrition Examination Survey. Endocr Abstr 2017;49:GP162.

27. Kang SY, Lim GE, Kim YK, Kim HW, Lee K, Park TJ, et al. Association between sarcopenic obesity and metabolic syndrome in postmenopausal women: a cross-sectional study based on the Korean National Health and Nutritional Examination Surveys from 2008 to 2011. J Bone Metab 2017;24:9-14.

28. Chung JY, Kang HT, Lee DC, Lee HR, Lee YJ. Body composition and its association with cardiometabolic risk factors in the elderly: a focus on sarcopenic obesity. Arch Gerontol Geriatr 2013;56:270-8.

29. Isanejad M, Mursu J, Sirola J, Kröger H, Rikkonen T, Tuppurainen $\mathrm{M}$, et al. Dietary protein intake is associated with better physical function and muscle strength among elderly women. Br J Nutr 2016;115:1281-91.

30. Kim JE, Lee YH, Huh JH, Kang DR, Rhee Y, Lim SK. Earlystage chronic kidney disease, insulin resistance, and osteoporosis as risk factors of sarcopenia in aged population: the fourth Korea National Health and Nutrition Examination Survey (KNHANES IV), 2008-2009. Osteoporos Int 2014;25:2189-98.

31. Buchmann N, Nikolov J, Spira D, Demuth I, Steinhagen-Thiessen E, Eckardt R, et al. Identifying sarcopenia in metabolic syndrome: data from the Berlin Aging Study II. J Gerontol A Biol Sci Med Sci 2016;71:265-72.

32. Ishii $S$, Tanaka $T$, Akishita $M$, Ouchi $Y$, Tuji T, Iijima K, et al. Metabolic syndrome, sarcopenia and role of sex and age: cross-sectional analysis of Kashiwa cohort study. PLoS One 2014;9:e112718.

33. Pereira FB, Leite AF, de Paula AP. Relationship between presarcopenia, sarcopenia and bone mineral density in elderly men. Arch Endocrinol Metab 2015;59:59-65.
34. Chalhoub D, Cawthon PM, Ensrud KE, Stefanick ML, Kado DM, Boudreau R, et al. Risk of nonspine fractures in older adults with sarcopenia, low bone mass, or both. J Am Geriatr Soc 2015;63:1733-40.

35. Lim S, Kim JH, Yoon JW, Kang SM, Choi SH, Park YJ, et al. Sarcopenic obesity: prevalence and association with metabolic syndrome in the Korean Longitudinal Study on Health and Aging (KLoSHA). Diabetes Care 2010;33:1652-4.

36. Baumgartner RN. Body composition in healthy aging. Ann N Y Acad Sci 2000;904:437-48.

37. Chin SO, Rhee SY, Chon S, Hwang YC, Jeong IK, Oh S, et al. Sarcopenia is independently associated with cardiovascular disease in older Korean adults: the Korea National Health and Nutrition Examination Survey (KNHANES) from 2009. PLoS One 2013;8:e60119.

38. Moon JH, Choo SR, Kim JS. Relationship between low muscle mass and metabolic syndrome in elderly people with normal body mass index. J Bone Metab 2015;22:99-106.

39. Han K, Park YM, Kwon HS, Ko SH, Lee SH, Yim HW, et al. Sarcopenia as a determinant of blood pressure in older Koreans: findings from the Korea National Health and Nutrition Examination Surveys (KNHANES) 2008-2010. PLoS One 2014;9:e86902.

40. Cooper H. Research synthesis and meta-analysis: a step-bystep approach. Thousand Oaks (CA): Sage Publications; 2015.

41. Du Y, No JK. Sarcopenia: nutrition and related diseases. Culinary Sci Hosp Res 2017;23:66-78.

42. Kim KS, Park KS, Kim MJ, Kim SK, Cho YW, Park SW. Type 2 diabetes is associated with low muscle mass in older adults. Geriatr Gerontol Int 2014;14 Suppl 1:115-21.

43. Perkisas S, Vandewoude M. Where frailty meets diabetes. Diabetes Metab Res Rev 2016;32 Suppl 1:261-7.

44. Park SH, Park JH, Song PS, Kim DK, Kim KH, Seol SH, et al. Sarcopenic obesity as an independent risk factor of hypertension. J Am Soc Hypertens 2013;7:420-5.

45. Aubertin-Leheudre M, Lord C, Goulet ED, Khalil A, Dionne IJ. Effect of sarcopenia on cardiovascular disease risk factors in obese postmenopausal women. Obesity (Silver Spring) 2006; 14:2277-83.

46. Du Y, Oh C, No JK. Osteosarcopenic obesity in elderly: the 
cascade of bone, muscle, and fat in inflammatory process. $\mathrm{Cu}$ linary Sci Hosp Res 2017;23:173-83.
47. Grimes DA, Schulz KF. Bias and causal associations in observational research. Lancet 2002;359:248-52. 